\title{
A new online monitoring application for high voltage switch based on non-contact current sensor
}

\author{
ZHAO Ke ${ }^{1}$, LI Yujie ${ }^{1, ~ *}$, ZHANG Liang ${ }^{1}$, CHEN Shaobo ${ }^{1}$, Wang Guoliang ${ }^{2}$, WANG Linjie ${ }^{2}$ \\ ${ }^{1}$ State Grid Jiangsu Electric Power Co. Ltd. Research Institute, Nanjing, 211103, China \\ ${ }^{2}$ State Grid Jiangsu Electric Power Co. Ltd. Maintenance Branch, Nanjing, 210006, China
}

\begin{abstract}
High voltage switches are indispensable in power system which will control and protect the high voltage lines. According to the research of high voltage switch equipment accident rate in the international conference on power grid for according, the high voltage switch equipment mechanical failure accidents accounted for more than $70 \%$. Thus, it is meaningful to research the switch monitoring technology and develop an integration device with easy installation and reliable data transmission. In this paper, a new type of non-contact current sensor is used to develop the online monitoring system for the mechanical characteristics of HV switches, which will monitor and analyze the action characteristics of the opening coil, closing coil and energy storage motor.
\end{abstract}

\section{INTRODUCTION}

High voltage switches are indispensable in power system which will control and protect the high voltage lines. Switches are used to on or off the circuit, especially when the system is in fault condition with large short-circuit current. Thus, the fault part will be quickly removed from power system to ensure the normal operation of the power system. In the primary equipment of the power system, the high-voltage switchgear is second in importance only to the transformer because of its quantity and task in the power system. The operation status of switches will directly affect the safety of the system. According to the research of high voltage switch equipment accident rate in the international conference on power grid for according, the high voltage switch equipment mechanical failure accidents accounted for more than 70\%[1-2].

At present, the power grid company has carried out condition-based maintenance for circuit breakers, which mainly has several problems: (1) The monitoring data is incomplete which will increase the cost of power cut. (2) Maintenance quality cannot be guaranteed. (3) The cost for state maintenance is high[3]. Therefore, it is meaningful to research the switch monitoring technology and develop an integration device with easy installation and reliable data transmission. This device can judge whether the technical parameters of the switch conform to the requirements of safety running. On the other hand, the characteristics of switches during operation can be monitored which will provide basic date for the power system maintenance and intelligent inspection [4]. Consequently, this device is of great significance by leading the development of the high voltage switch equipment state fusion perception technology, ensuring the reliability of power system operation, reducing the loss caused by switch failure.

In this paper, a new type of non-contact current sensor is used to develop the online monitoring system for the mechanical characteristics of $\mathrm{HV}$ switches, which will monitor and analyze the action characteristics of the opening coil, closing coil and energy storage motor.

\section{COIL CURRENT CHARACTERISTIC ANALYSIS}

\subsection{Opening coil and closing coil}

The opening and closing action of switches is triggered by the magnetic force of the iron core. Since the iron core will be subjected to different resistance in different stages while moving, the coil current will be segmented and constantly changing. When the iron core and its moving mechanism have novel conditions, such as stuck, trip, rejection, etc., the coil current waveform will be novel. So it can analyze the operation state of the switch by completing the recording and analysis of the coil current waveform in the whole process of the coil action [5-6]. The typical switching current waveform under normal working conditions is shown in figure 1(a).

The $t_{0}-t_{1}$ section is the initial energizing phase while the coil current rises exponentially and the core is at rest. This stage is determined by the coil resistance and control power supply, which can reflect the state of the coil. The $\mathrm{t}_{1}-\mathrm{t}_{2}$ section is the initial movement stage. When the iron core starts to move, it does not touch the closing and closing thimble. At this point, the counter electromotive force has a small effect, increasing the speed of the iron core and decreasing the current value. According to this waveform of this section, we can judge whether there is

\footnotetext{
*Corresponding author: 1275911032@qq.com
} 
tripping and sticking in the process of core movement. The $t_{2}-t_{3}$ stage is the action section, when the iron core touches the thimble, the force on the iron core increases, and the current value increases, so it can be judged whether the thimble movement mechanism is novel. At $t_{3}$, the core stops moving. The $t_{4}-t_{5}$ section is the recovery section, when the switch completes the action, an arc will be generated between the contacts. As the arc voltage keeps rising, the coil current drops rapidly [7].

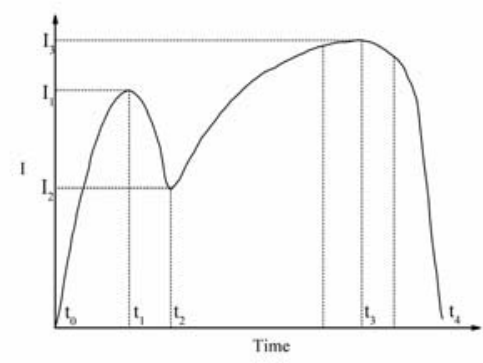

(a) Opening and Closing Coil

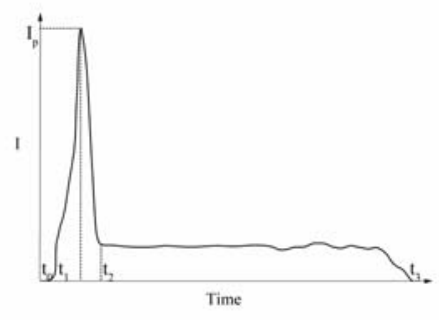

(b) Energy Storage Motor

Fig. 1 Current Waveform

\subsection{Energy storage motor}

A spring is used for energy storage for the breaker with the spring operating mechanism. The spring begins to store the energy for the opening and closing action when the energy storage circuit is closed after the closing action of the switch [8]. The current waveform of the energy storage motor is shown in figure 1(b). At $T_{1}$, the energy storage motor starts without load. At time $\mathrm{T}_{2}$, the motor rotates without load. This is because the electrical energy of the energy storage motor has not been converted into the mechanical energy of the spring mechanism, and the current starts to stabilize from $\mathrm{T}_{2}$. At $\mathrm{T}_{3}$, the energy storage motor starts to release energy and pulls the spring to work. The stiffness state of the spring and the lubrication degree of the energy storage shaft can be obtained from this waveform.

\section{DETAIL DESIGN}

\subsection{Overall architecture}

The online monitoring application for switch mechanical characteristics is consisted of three different range current sensors and a controller. The controller has the function of triggering so that the real-time collection and transmission of waveform can be completed while the switch acting. All data will be transmitted to the analysis system placed in station or cloud by wireless. The analysis system will complete the storage, display and analysis of the switching characteristics of the whole station. The monitoring architecture of a single circuit breaker is shown in the figure 2(a), and the monitoring topology of the whole station is shown in the figure 2(b). The application in this paper was developed based on a $10 \mathrm{kV}$ vacuum circuit breaker.

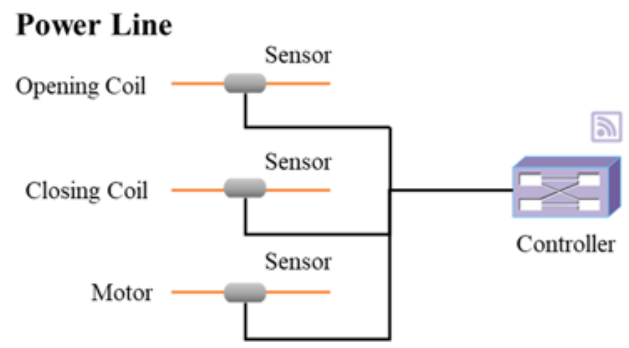

(a) Single Breaker

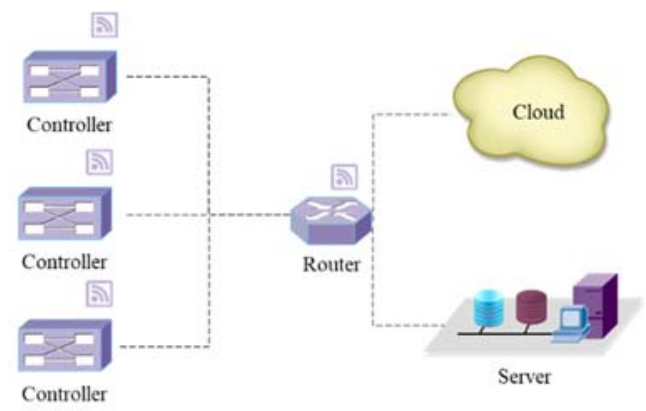

(b) Whole Station

Fig. 2 Monitoring Topology

\subsection{Design of current sensors}

The current sensor for online monitoring needs to be used for a long time without damaging the stability of the original high voltage switch. Therefore, the current should be measured in a non-contact and non-destructive way. At the same time, because of the narrow space in the high voltage switch, the sensor structure design should be as small as possible to install easily.

The hall current sensor can measure the current of the wire without contact, which can be used in the monitoring application in this paper. When a magnetic field with magnetic induction intensity $\mathrm{B}$ is applied to the normal direction of the hall element plane, an electric potential Vh will be generated between the direction perpendicular to the current and the magnetic field (i.e., the hall output). It is called hall potential, and its size is proportional to the magnetic field $\mathrm{B}$. Thus, there is a corresponding relationship between I and $\mathrm{B}$. Therefore, the potential output of the hall current sensor has a linear relationship with the wire current.

The circuit of the current sensor is shown in figure 5 . A wire is used to connect the sensor to the controller. The wire is divided into 2 channels, one channel is the power supply for the sensor, and the other is the output of hall potential. For the circuit breaker in this paper, through long-term test, the current peak of the opening and closing 
coil is 6A, and the current peak of the energy storage motor is $3 \mathrm{~A}$. Therefore, the range of the current sensor for opening and closing current is $10 \mathrm{~A}$, and the range of the current sensor for energy storage motor is $5 \mathrm{~A}$.

The sensor is designed in the form of a ring with wires passing through, so that sensors can be conveniently installed. The inside diameter of the sensor is $3 \mathrm{~mm}$ according to the cross-sectional area of the conductor which is $1.5 \mathrm{~m}^{2}$. The outside diameter of the sensor is $12 \mathrm{~mm}$, and the length is $20 \mathrm{~mm}$. Its structure is shown in figure 3 . The size is small and will be install easily.
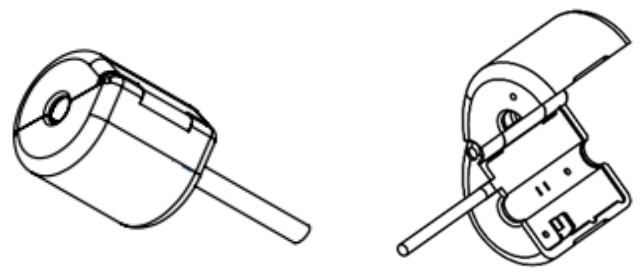

Fig. 3 Structure of Sensor

\subsection{Design of controller}

The controller is the core of the monitoring application, which has the functions of signal triggering, waveform recording and waveform transmission. The basic architecture is shown in figure 6.

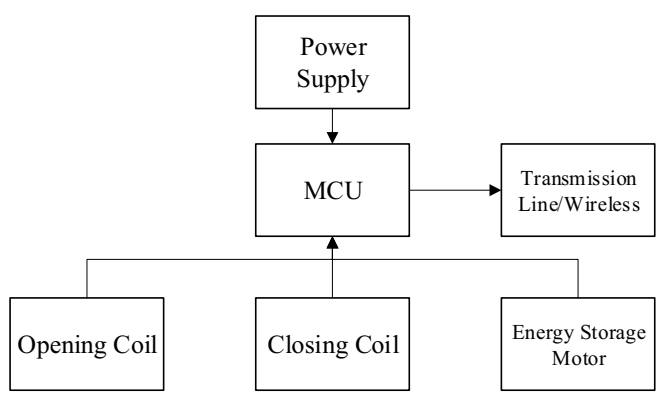

Fig. 4 Architecture of Controller

The data acquisition modules of the opening coil, closing coil and energy storage motor are the same. The signals enter the MCU through amplifier and $\mathrm{AD}$, and the MCU will monitor the changes of current value in real time. When the breaker is not in operation, the current value detected by each current sensor is zero, and the MCU can only receive the zero value returned by the sensor. The single signal circuit is shown in figure 5(a). In this design, the XX model was selected for $\mathrm{AD}$, the sampling rate was set to $5 \mathrm{kHz}$, and the sampling accuracy was 14bit. Through the high-precision amplifier and AD system, the current accuracy of non-contact measurement could reach $1 \%$.

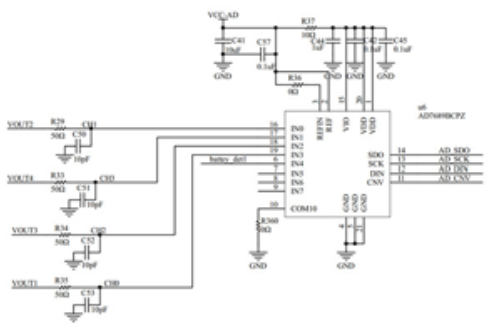

(a) Signal

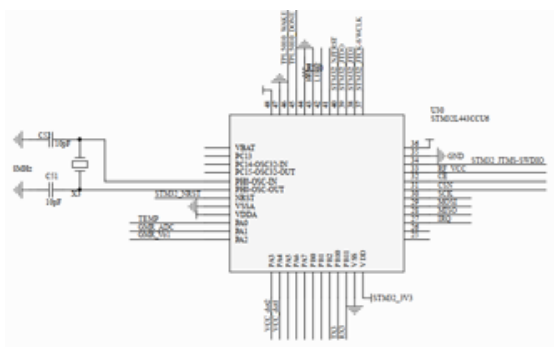

(b) $\mathrm{MCU}$

Fig. 5 Circuit of Controller

The MCU is STM32L443CCU6 with the highest main frequency up to $168 \mathrm{MHz}$. The operating voltage is 1.8 to $3.6 \mathrm{~V}$. The standby power consumption of the MCU is low, and it has abundant interfaces, which can realize the transmission of multi-channel data and facilitate the expansion. The circuit of the MCU is shown in figure 5 (b). The main controller realizes the monitoring of three loop signals through software triggering, and its working logic is shown in figure 6 . All three signals are monitored independently. In a signal action, independent monitoring can ensure the triggering quickly so that it can monitor fast reclosing action.

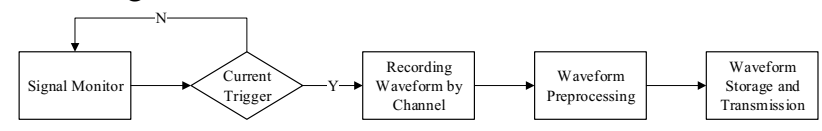

Fig. 6 Working Logic of Controller

The controller provides wired and wireless data transmission. Wired mode is transmitted through network cable, while wireless mode is transmitted through $2.4 \mathrm{G}$. The communication distance of $2.4 \mathrm{G}$ can reach as far as $200 \mathrm{~m}$, and its data transmission rate can reach $2 \mathrm{Mbit} / \mathrm{s}$. Therefore, a single point can collect date from all monitoring applications in one station. All current waveform data will be transmitted in COMTRADE format for data analysis.

\subsection{Design of monitoring system}

The online monitoring system can be deployed in the substation to collect, store, analyze and query the monitoring data of all switch mechanical characteristics. The analysis principle of the operation characteristics of the coils and the energy storage motor is shown in the second section of this paper.

For opening and closing coil, characteristics can be calculated through waveform, such as wave duration $\mathrm{T}_{5}$, electric shock contact time $T_{2}$, peak current $I_{3}$ and peak current $I_{1}$. The four values are within a reasonable range for the same type of breaker. Operators can get early warning of coils from the running state when these values are out of range.

For the energy storage motor current, the fault judgment method is the same as coils with the values such as time $T_{2}$, wave duration $T_{5}$ and peak current $I_{P}$. In the next step, the neural network algorithm can be introduced to automatically judge the fault by using the algorithm to automatically learn the threshold range and reasonable working range for different types of switching equipment. 


\section{TEST}

In order to verify the function of the online monitoring system for switches, a test platform was constructed in the laboratory with a $10 \mathrm{kV}$ circuit breaker. The sensors developed in this paper is installed in the circuit breaker's coils and energy storage motor circuit. All the data processing is realized through the controller and transmitted to the main station through the network cable. The monitoring performance of the device was tested in the long-term test. After 15,000 times of operation, it can be found that the opening and closing coil wave duration T5 increases significantly, which indicates that the opening and closing time of the breaker is significantly increased due to mechanical strain during the opening and closing process.

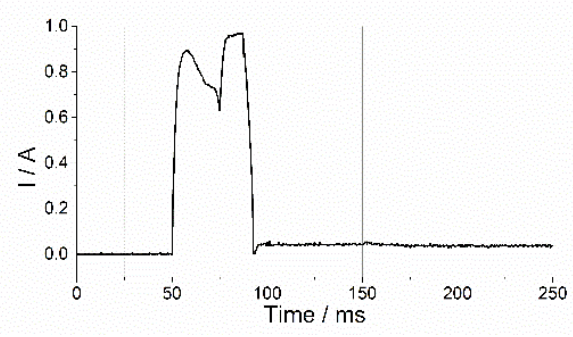

Fig. 7 Waveform of closing coil

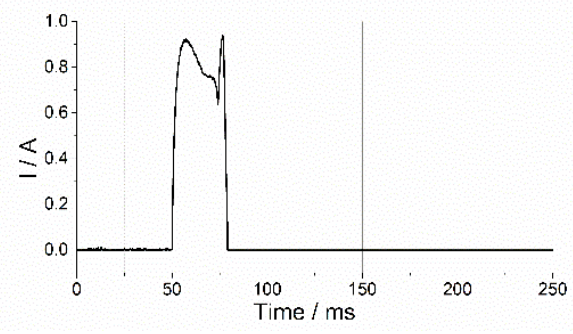

Fig. 8 Waveform of opening coil

\section{CONCLUSION}

In this paper, a new type of non-contact current sensor is used to develop the online monitoring system for the mechanical characteristics of HV switches, which will monitor and analyze the action characteristics of the opening coil, closing coil and energy storage motor. By analyzing the characteristics of the current waveform of the coils and motor, a new contactless current sensor is developed. The operation condition monitoring and early warning of high-voltage switch are realized by calculating the characteristic value of waveform. The system has been tested for a long time in the laboratory, which meets the design.

\section{Reference}

1. XIE Jiazheng, XIA Chengjun, MA Zhongneng. Research on Cost Sensitivity and Cost Estimation Model of Low-voltage Switchgear[J]. Electric Power Engineering Technology, 2017,36(05):81-85+92.
2. ZHAO Guodong, GENG Yaming, CHAI Yu, et al. The Applications of the Optical Fiber Displacement Sensor in On-line Monitoring System of Circuit Breaker[J]. Electric Power Engineering Technology, 2017, 36(04):98-102.

3. HUANG Xinbo, TAO Chen, LIU Bin. On-line Monitoring Technology of Mechanical Properties and State Evaluation Method for Intelligent Circuit Breaker. High Voltage Apparatus. 2015 Vol.51: 129 134.

4. MEI, Fei, et al. Development and application of distributed multilayer on-line monitoring system for high voltage vacuum circuit breaker. Journal of Electrical Engineering and Technology, 2013, 8.4: 813-823.

5. TIAN Tao, ZHANG Zhaojun, ZHU Chao, et al. Reliability and Life Analysis of Energy-storing Spring for High Voltage Circuit Breaker[J]. Electric Power Engineering Technology, 2017,36(05):103108.

6. YAN, Lu; JIAN-WEI, Shen. Study on Data FusionBased Online Monitoring System for Mechanical Characteristics of Circuit Breaker. International Journal of Hybrid Information Technology, 2016, 9.12: 141-152.

7. YE Yuyuan, SHA Haoyuan, LIANG Junhan, et al. Defect Diagnosis Technology of Operating Mechanism of Circuit Breakers Based on Wavelet Packet Energy $[\mathrm{J}]$. Electric Power Engineering Technology, 2018, 37(04): 71-77.

8. LIANG Junhan, ZHENG Jianyong, PAN Yi. On-line Monitoring System Design of Circuit Breaker Based on Multi-core Structure $[\mathrm{J}]$. Electric Power Engineering Technology, 2017, 36(06): 68-72+77. 